\section{Reply to "Need for further research on the freshwater fish fauna of the Ashambu Hills landscape: a response to Abraham et al."}

\section{Robin Kurian Abraham ${ }^{1}$, Nachiket Kelkar ${ }^{2}$ \& A. Biju Kumar ${ }^{3}$}

1,2 TC 11/1123, YMR Junction, Kowdiar P.O., Nanthencode, Thiruvananthapuram, Kerala 695003, India

${ }^{3}$ Department of Aquatic Biology and Fisheries, University of Kerala, Kariavattom, Thiruvananthapuram, Kerala 695581, India Email: ${ }^{1}$ robinabrahamf50@gmail.com, ${ }^{2}$ rainmaker.nsk@gmail. com (corresponding author), ${ }^{3}$ bijupuzhayoram@gmail.com

The response to the article and checklist (Abraham et al. 2011) by Raghavan (2011) is timely, and much appreciated. Such critical reading of manuscripts would not only help the authors to prepare the manuscripts with caution but aid fish taxonomists and researchers planning to work on similar topics. The critique has rightfully pointed out a few shortcomings that we overlooked. We are grateful to some of the constructive suggestions in the critical response, as this was a primary attempt to prepare a comprehensive database of fishes in the west-flowing drainages of the Ashambu Hills. We provide in this reply, a revised checklist for freshwater fishes in this region, based on some of the respondent's suggestions.

We thank the respondent for pointing out some references we inadvertently overlooked (e.g. Kurup et al. 2004). We also missed some species from the list, largely due to taxonomic ambiguities or unavailability of obscure references (e.g. Jerdon 1849; Arnold 1911; Euphrasia et al. 2006) to us. Further, we had also decided to have strict criteria for including references that were published in journals or as theses (compiling

Date of publication (online): 26 May 2011

Date of publication (print): 26 May 2011

ISSN 0974-7907 (online) | 0974-7893 (print)

Manuscript details:

Ms \# 02797

Received 05 May 2011

Citation: Abraham, R.K., N. Kelkar \& A.B. Kumar (2011). Reply to "Need for further research on the freshwater fish fauna of the Ashambu Hills landscape: a response to Abraham et al.". Journal of Threatened Taxa 3(5): 1792-1797.

Copyright: @ Robin Kurian Abraham, Nachiket Kelkar \& A. Biju Kumar 2011. Creative Commons Attribution 3.0 Unported License. JoTT allows unrestricted use of this article in any medium for non-profit purposes, reproduction and distribution by providing adequate credit to the authors and the source of publication.

OPEN ACCESS | FREE DOWNLOAD individual papers by authors from the theses). This was done because conference proceedings were often confusing for proper

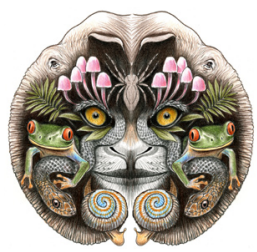
citation as their publication info was inadequate, leading to certain key omissions, as pointed. Finally, some references mentioned in the critique are rather new, (ongoing doctoral research cited in the response; Eschmeyer \& Fricke 2011) and we would like to request the consideration that our manuscript was submitted before these publications, so some references may have been overlooked in the final version too.

Also, we would like to discuss the taxonomic status and occurrence of some species as it appears in our paper, with the following clarifications:

(i) The 'missing' species highlighted by the critique such as Hypselobarbus thomassi, Tor khudree, Botia striata, Nemacheilus guentheri, Mystus sengtee, Glyptothorax lonah and Mystus gulio are indeed species that were not included in the checklist, because of doubts about the taxonomic status of these species, and we did not describe in detail within the paper.

(ii) Also, many previous checklists that were consulted were seen to repeat earlier ones, apparently without extensive fieldwork, as remarked by the critique. Moreover, in our field sampling we did not find some species mentioned in earlier checklists, such as Barilius gatensis, which have been shown to be abundant in all the sampled rivers by past authors, hence the omission of some species in our paper.

(iii) The status of Puntius melanampyx has been ambiguous in literature and synonymized with $P$. fasciatus in earlier literature (Jayaram 1991, 2010). We add a new species Puntius kannikattiensis in our checklist. We sampled this species in Neyyar and Karamana rivers (reported from KMTR by Arunachalam \& Johnson 2002).

(iv) With regard to our usage of the term, 'range extension', even though we have not explicitly used it on individual rivers, we have extended the ranges of some of the species, towards the south by a river or two. Garra hughi, as we mentioned in our paper, had been reported from the headwaters of the Vamanapuram River by Johnson \& Arunachalam (2010). But, our study reports a population further south into the Neyyar River. And our goal is not to merely mention the novel southernmost range for the species, but to elaborate on 
the occurrence of the species in all drainages sampled, keeping in mind that such information would be of interest to any biogeographic work.

(v) Puntius mahecola, similarly, reported by Pethiyagoda \& Kottelat (2005) to occur in Kallada, was only mentioned and indicated in the map to provide a wholesome representation of the species distribution in the sampled landscape. We have recorded it from all five sampled rivers (with the rivers Karamana and Neyyar being previously not reported), and hence a range extension for the species. It may be noted that the title of our paper is not 'range extension into the Ashambu Hills'. We also agree that Puntius mahecola is not the synonym of $P$. amphibius and the taxonomic ambiguities remain to be resolved as to what actually represents the latter species, leaving scope for more comprehensive work, especially including the type localities.

(vi) Taxonomic ambiguities with regard to Garra mcclellandi and G. periyarensis remain to be resolved and further research, incorporating molecular taxonomy, would help resolve these issues. At present we believe the specimen we have is G. mcclellandi. As our studies are ongoing, voucher specimens will be made available for scrutiny soon.

(vii) Additionally, further studies are warranted to record the population status of Hypselobarbus thomassi in the Kallada River system. If, as the critique mentions, that studies are being currently undertaken for the same, then that should help resolve any taxonomic misunderstandings for the species in this region. Eschmeyer \& Fong (2010) treat Tor khudree malabaricus as T. malabaricus; so the species we refer to is synonymous with T. khudree.

Finally, we would like to reiterate that the primary intention and scope of our paper was to present a checklist of fish species occurring in the west-flowing rivers of the Ashambu Hills of Kerala and not provide a comprehensive taxonomic treatment as such. We would also like to mention here that some taxonomic limitations of the study arise from the minimally invasive sampling approaches we preferred to use, whereby we did not make excessive 'collections' of every sampled species. We did make specimen

Table 1. An annotated, revised checklist of freshwater fish species known from the Ashambu Hills landscape. This checklist is derived from previous literature (see above) and updated by species sampled during our study (species for which preferred habitat, elevation range and occurrence are mentioned).

\begin{tabular}{|c|c|c|c|c|c|}
\hline Genus & Species Author & Threats & $\begin{array}{c}\text { Preferred } \\
\text { Habitat }\end{array}$ & $\begin{array}{c}\text { Elevation } \\
\text { Range }\end{array}$ & Occurrence in Rivers \\
\hline \multicolumn{6}{|l|}{ Ambassidae } \\
\hline Chanda & nama Hamilton & $\mathrm{HL}$ & $\mathrm{Ru}$ & $\mathrm{m}$ & KLD \\
\hline Parambassis & dayi ${ }^{+}$Bleeker & $\mathrm{HL}$ & & & \\
\hline Parambassis & thomassi $^{+}$(Day) & HL, DY, IN & $\mathrm{Ru}$ & $\mathrm{m}$ & KLD, VAM, KAR, NEY \\
\hline Pseudambassis & ranga (Hamilton-Buchanan) & $\mathrm{HL}$ & $\mathrm{Ru}$ & $\mathrm{m}$ & KLD, KAR, NEY \\
\hline \multicolumn{6}{|l|}{ Anabantidae } \\
\hline Anabas & testudineus Bloch & $\mathrm{HL}, \mathrm{IN}, \mathrm{DY}$ & & & \\
\hline \multicolumn{6}{|l|}{ Anguillidae } \\
\hline Anguilla & bengalensis Gray & $\mathrm{HL}$ & $\mathrm{Ru}, \mathrm{PI}$ & $\mathrm{I}, \mathrm{m}, \mathrm{h}$ & KLD, ITK, VAM, KAR, NEY \\
\hline Anguilla & bicolor McClelland & $\mathrm{HL}$ & & & \\
\hline \multicolumn{6}{|l|}{ Aplocheilidae } \\
\hline Aplocheilus & lineatus (Valenciennes) & $\mathrm{HL}$ & $\mathrm{PI}$ & $\mathrm{I}, \mathrm{m}$ & KLD, ITK, VAM, KAR, NEY \\
\hline Aplocheilus & blockii (Arnold) & $\mathrm{HL}$ & $\mathrm{PI}$ & $\mathrm{m}$ & NEY \\
\hline \multicolumn{6}{|l|}{ Bagridae } \\
\hline Batasio & travancorica + Hora \& Law & $\mathrm{HL}, \mathrm{DY}$ & & & \\
\hline Horabagrus & brachysoma + (Gunther) & HL, DY & $\mathrm{PI}$ & $\mathrm{I}, \mathrm{m}$ & KLD, KAR, NEY \\
\hline Mystus & armatus Day & $\mathrm{HL}$ & & & \\
\hline Mystus & bleekeri (Day) & $\mathrm{HL}$ & $\mathrm{PI}$ & $\mathrm{m}, \mathrm{h}$ & NEY \\
\hline Mystus & gulio (Hamilton) & & & & \\
\hline
\end{tabular}




\begin{tabular}{|c|c|c|c|c|c|}
\hline Genus & Species Author & Threats & $\begin{array}{c}\text { Preferred } \\
\text { Habitat }\end{array}$ & $\begin{array}{c}\text { Elevation } \\
\text { Range }\end{array}$ & Occurrence in Rivers \\
\hline Mystus & keletius (Valenciennes) & $\mathrm{HL}$ & & & \\
\hline Mystus & malabaricus + (Jerdon) & HL, DY & $\mathrm{PI}$ & $\mathrm{m}, \mathrm{h}$ & KLD, ITK, VAM, KAR, NEY \\
\hline Mystus & montanus Jerdon & $\mathrm{HL}$ & & & \\
\hline Mystus & oculatus Valenciennes & $\mathrm{HL}$ & $\mathrm{Ru}, \mathrm{PI}$ & $\mathrm{m}$ & NEY \\
\hline Mystus & sengtee Hamilton-Buchanan & $\mathrm{HL}$ & & & \\
\hline Mystus & vittatus Bloch & $\mathrm{HL}$ & & & \\
\hline \multicolumn{6}{|l|}{ Balitoridae } \\
\hline Bhavania & australis + Jerdon & $\mathrm{HL}$ & $\mathrm{Ra}$ & $\mathrm{h}$ & KLD \\
\hline Travancoria & jonesi ${ }^{+}$Hora & $\mathrm{HL}$ & $\mathrm{Ra}, \mathrm{Ri}$ & $\mathrm{h}$ & KLD, VAM \\
\hline Nemacheilus & denisoni ${ }^{+}$Day & $\mathrm{HL}$ & & & \\
\hline Nemacheilus & pulchellus + Day & $\mathrm{HL}$ & & & \\
\hline Nemacheilus & guentheri + Day & $\mathrm{HL}$ & & & \\
\hline Nemacheilus & triangularis + Day & $\mathrm{HL}$ & $\mathrm{Ra}, \mathrm{Ru}, \mathrm{Ri}$ & $\mathrm{m}, \mathrm{h}$ & KLD, VAM, KAR, NEY \\
\hline Pangio & goaensis ${ }^{+}$Tilak & $\mathrm{HL}$ & & & \\
\hline Lepidocephalichthys & thermalis (Valenciennes) & $\mathrm{HL}$ & $\mathrm{Ra}, \mathrm{Ru}, \mathrm{Ri}$ & $\mathrm{m}, \mathrm{h}$ & KLD \\
\hline Xenentodon & cancilla Hamilton-Buchanan & HL, DY & $\mathrm{Ru}, \mathrm{PI}$ & $\mathrm{I}, \mathrm{m}$ & KLD, ITK, VAM, KAR, NEY \\
\hline \multicolumn{6}{|l|}{ Channidae } \\
\hline Channa & gachua Bloch \& Schneider & HL, DY, IN & $\mathrm{Ru}, \mathrm{PI}$ & $\mathrm{I}, \mathrm{m}$ & VAM \\
\hline Channa & marulius Hamilton-Buchanan & HL, DY, IN & $\mathrm{Ru}, \mathrm{PI}$ & $\mathrm{I}, \mathrm{m}$ & VAM, KLD \\
\hline Channa & striata (Bloch) & HL, DY & $\mathrm{Ru}, \mathrm{PI}$ & $\mathrm{I}, \mathrm{m}$ & KLD, ITK, VAM, KAR, NEY \\
\hline Channa & diplogramma +,^ (Day) & HL, DY, OF & $\mathrm{Ru}, \mathrm{PI}$ & $\mathrm{m}$ & KLD \\
\hline \multicolumn{6}{|l|}{ Cichlidae } \\
\hline Etroplus & maculatus (Bloch) & HL, DY & $\mathrm{Ru}, \mathrm{PI}$ & $\mathrm{I}, \mathrm{m}$ & KLD, ITK, VAM, KAR, NEY \\
\hline Etroplus & suratensis (Bloch) & $\mathrm{HL}, \mathrm{DY}$ & $\mathrm{Ru}, \mathrm{PI}$ & 1 & KLD, ITK, VAM, KAR, NEY \\
\hline Oreochromis & mossambicus (Peters) & - & $\mathrm{Ru}, \mathrm{PI}$ & $\mathrm{I}, \mathrm{m}$ & NEY, KLD \\
\hline \multicolumn{6}{|l|}{ Clariidae } \\
\hline Clarias & dussumieri + Valenciennes & HL, OF, IN & $\mathrm{PI}$ & $\mathrm{m}$ & KLD, NEY \\
\hline Heteropneustes & fossilis Bloch & HL, DY & $\mathrm{PI}$ & $\mathrm{I}, \mathrm{m}$ & NEY \\
\hline \multicolumn{6}{|l|}{ Clupeidae } \\
\hline Dayella & malabarica + (Day) & $\mathrm{HL}$ & $\mathrm{Ru}$ & $\mathrm{m}$ & KLD \\
\hline \multicolumn{6}{|l|}{ Cyprinidae } \\
\hline Laubuca & dadyburjori + Menon & $\mathrm{HL}$ & & & \\
\hline Salmophasia & boopis + Day & $\mathrm{HL}, \mathrm{DY}$ & & & \\
\hline Salmophasia & balookee (Sykes) & $\mathrm{HL}, \mathrm{DY}$ & $\mathrm{Ru}$ & $\mathrm{m}$ & NEY \\
\hline Esomus & danricus Hamilton-Buchanan & & & & \\
\hline Esomus & thermoicos Valenciennes & & & & \\
\hline Devario & aequipinnatus (McClelland) & $\mathrm{HL}$ & $\mathrm{Ru}, \mathrm{PI}$ & $\mathrm{I}, \mathrm{m}, \mathrm{h}$ & KLD, ITK, VAM, KAR, NEY \\
\hline Devario & malabaricus + (Jerdon) & $\mathrm{HL}$ & $\mathrm{Ru}, \mathrm{PI}$ & $\mathrm{I}, \mathrm{m}, \mathrm{h}$ & KLD, ITK, VAM, KAR, NEY \\
\hline Rasbora & daniconius (Hamilton) & $\mathrm{HL}$ & $\mathrm{Ru}, \mathrm{PI}$ & $\mathrm{I}, \mathrm{m}, \mathrm{h}$ & KLD, ITK, VAM, KAR, NEY \\
\hline Amblypharyngodon & melettinus (Valenciennes) & $\mathrm{HL}, \mathrm{DY}$ & $\mathrm{Ru}$ & $\mathrm{I}, \mathrm{m}$ & NEY \\
\hline Amblypharyngodon & microlepis (Bleeker) & $\mathrm{HL}$ & & & \\
\hline Barilius & bakeri+Day & $\mathrm{HL}, \mathrm{EX}$ & $\mathrm{Ra}, \mathrm{Ru}$ & $\mathrm{m}, \mathrm{h}$ & KLD, ITK, VAM, KAR, NEY \\
\hline Barilius & bendelisis Hamilton-Buchanan & $\mathrm{HL}$ & & & \\
\hline Barilius & gatensis ${ }^{+}$Valenciennes & $\mathrm{HL}$ & & & \\
\hline Cyprinus & carpio Linnaeus & - & $\mathrm{PI}$ & $\mathrm{m}$ & KLD, NEY \\
\hline Ctenopharyngodon & idella Valenciennes & - & & & \\
\hline
\end{tabular}




\begin{tabular}{|c|c|c|c|c|c|}
\hline Genus & Species Author & Threats & $\begin{array}{c}\text { Preferred } \\
\text { Habitat }\end{array}$ & $\begin{array}{c}\text { Elevation } \\
\text { Range }\end{array}$ & Occurrence in Rivers \\
\hline Labeo & dussumieri ${ }^{+}$Valenciennes & HL, EX & & & \\
\hline Labeo & rohita Hamilton-Buchanan & $\mathrm{HL}$ & & & \\
\hline Labeo & calbasu Hamilton-Buchanan & $\mathrm{HL}$ & & & \\
\hline Tor & malabaricus $^{+}$(Jerdon) & HL, OF, DY, EX & $\mathrm{PI}$ & $\mathrm{m}, \mathrm{h}$ & KLD, VAM, KAR, NEY \\
\hline Catla & catla Valenciennes & - & & & \\
\hline Cirrhinus & mrigala Hamilton-Buchanan & - & & & \\
\hline Garra & mcclellandi ${ }^{+,}$RE (Jerdon) & HL, DY, EX & $\mathrm{Ra}, \mathrm{Ru}$ & $\mathrm{h}$ & NEY \\
\hline Garra & mullya (Sykes) & HL, DY, EX & $\begin{array}{c}\text { Ra, Ru, } \\
\text { PI, Ri }\end{array}$ & $\mathrm{l}, \mathrm{m}, \mathrm{h}$ & KLD, ITK, VAM, KAR, NEY \\
\hline Garra & hughi $^{+,}$RE Silas & $\mathrm{HL}$ & $\begin{array}{l}\mathrm{Ra}, \mathrm{Ru} \\
\mathrm{PI}, \mathrm{Ri}\end{array}$ & $\mathrm{h}$ & KLD, VAM, NEY \\
\hline Garra & $\begin{array}{l}\text { surendranathanii }{ }^{+} \\
\text {Shaji, Arun \& Easa }\end{array}$ & $\mathrm{HL}$ & & & \\
\hline Horalabiosa & joshuai ${ }^{+}$Silas & $H L, E X$ & & & \\
\hline Hypselobarbus & curmuca + (Hamilton) & $\begin{array}{l}\text { HL, OF, DY, } \\
\text { EX, IN }\end{array}$ & $\mathrm{Ru}, \mathrm{PI}$ & $\mathrm{m}, \mathrm{h}$ & KLD, ITK, VAM, KAR, NEY \\
\hline Hypselobarbus & jerdoni + RE (Day) & HL, EX, DY & $\mathrm{Ru}, \mathrm{PI}$ & $\mathrm{m}$ & KLD \\
\hline Hypselobarbus & kolus $^{+}$(Sykes) & HL, DY & $\mathrm{Ru}, \mathrm{PI}$ & $\mathrm{m}$ & KLD \\
\hline Hypselobarbus & kurali $^{+}$Menon \& Rema Devi & $\mathrm{HL}, \mathrm{DY}$ & $\mathrm{Ru}, \mathrm{PI}$ & $\mathrm{m}$ & KLD \\
\hline Hypselobarbus & thomassi & & & & \\
\hline Osteobrama & bakeri+ Day & HL, DY, IN & $\mathrm{Ru}, \mathrm{PI}$ & $\mathrm{m}$ & KLD \\
\hline Puntius & arulius Jerdon & $\mathrm{HL}$ & & & \\
\hline Puntius & bimaculatus (Bleeker) & HL, DY, IN & & & \\
\hline Barbodes & carnaticus $^{+}$(Jerdon $)$ & $\mathrm{HL}$ & & & \\
\hline Puntius & chola Hamilton-Buchanan & $\mathrm{HL}, \mathrm{DY}, \mathrm{IN}$ & & & \\
\hline Puntius & conchonius Hamilton-Buchanan & HL, DY, IN & & & \\
\hline Puntius & denisonii + Day & HL, DY, OF & & & \\
\hline Puntius & dorsalis (Jerdon) & HL, DY & $\mathrm{Ru}, \mathrm{PI}$ & $\mathrm{m}, \mathrm{h}$ & KLD, ITK, VAM, NEY \\
\hline Puntius & $\begin{array}{l}\text { exclamatio }{ }^{+,} \text {ASH Pethiyagoda \& } \\
\text { Kottelat }\end{array}$ & HL, EX, DY & $\mathrm{Ru}, \mathrm{PI}$ & $\mathrm{m}$ & KLD \\
\hline Puntius & fasciatus ${ }^{+}$(Jerdon) & $H L, D Y$ & $\mathrm{Ru}, \mathrm{PI}, \mathrm{Ri}$ & $\mathrm{m}, \mathrm{h}$ & KLD, VAM, KAR, NEY \\
\hline Puntius & filamentosus (Valenciennes) & HL, DY, IN & $\mathrm{Ru}, \mathrm{PI}$ & $\mathrm{m}$ & KLD, ITK, VAM, KAR, NEY \\
\hline Puntius & $\begin{array}{l}\text { kannikattiensis+ (Arunachalam \& } \\
\text { Johnson, 2002) }\end{array}$ & HL, DY & $\mathrm{Ru}, \mathrm{Pl}, \mathrm{Ri}$ & $\mathrm{h}$ & NEY, KAR \\
\hline Puntius & sp. nov ${ }^{+, \#, A S H}$ & $\mathrm{HL}$ & $\mathrm{Ru}, \mathrm{PI}$ & $\mathrm{m}$ & ITK \\
\hline Puntius & mahecola $^{+,}$RE (Valenciennes) & $\mathrm{HL}, \mathrm{IN}$ & $\mathrm{Ru}, \mathrm{PI}$ & $\mathrm{m}$ & ITK, NEY \\
\hline Puntius & parrah Day & $\mathrm{HL}$ & $\mathrm{Ru}, \mathrm{PI}$ & $\mathrm{m}$ & KAR \\
\hline Puntius & sarana subnasutus + Valenciennes & HL, OF, IN, DY & $\mathrm{Ru}, \mathrm{PI}$ & $\mathrm{I}, \mathrm{m}$ & KLD, ITK, NEY \\
\hline Puntius & tambraparniei ${ }^{+}$Silas & $\mathrm{HL}$ & & & \\
\hline Puntius & ticto Hamilton-Buchanan & HL, EX, DY, IN & $\mathrm{Ru}, \mathrm{PI}, \mathrm{Ri}$ & $\mathrm{m}$ & KLD, ITK, KAR, NEY \\
\hline Puntius & vittatus Day & $\mathrm{HL}$ & & & \\
\hline \multicolumn{6}{|l|}{ Gobiidae } \\
\hline Sicyopterus & griseus Day & $\mathrm{HL}$ & $\mathrm{Ru}$ & $\mathrm{I}, \mathrm{m}$ & KAR \\
\hline Awaous & gutum Hamilton-Buchanan & $\mathrm{HL}$ & $\mathrm{Ru}$ & $\mathrm{I}, \mathrm{m}$ & KAR \\
\hline Glossogobius & giuris Hamilton-Buchanan & $\mathrm{HL}, \mathrm{DY}, \mathrm{IN}$ & $\mathrm{Ru}$ & I, m & KLD, ITK, VAM, KAR \\
\hline \multicolumn{6}{|l|}{ Hemiramphidae } \\
\hline Hyporamphus & limbatus Valenciennes & $\mathrm{HL}$ & $\mathrm{Ru}, \mathrm{PI}$ & 1 & KLD \\
\hline \multicolumn{6}{|l|}{ Mastacembelidae } \\
\hline Mastacembelus & armatus (Lacepede) & HL, OF, DY, IN & $\mathrm{Ru}, \mathrm{Pl}, \mathrm{Ri}$ & $\mathrm{l}, \mathrm{m}, \mathrm{h}$ & KLD, NEY \\
\hline
\end{tabular}




\begin{tabular}{|c|c|c|c|c|c|}
\hline Genus & Species Author & Threats & $\begin{array}{c}\text { Preferred } \\
\text { Habitat }\end{array}$ & $\begin{array}{c}\text { Elevation } \\
\text { Range }\end{array}$ & Occurrence in Rivers \\
\hline Macrognathus & guentheri (Day) & $\mathrm{HL}, \mathrm{DY}$ & & & \\
\hline \multicolumn{6}{|l|}{ Nandidae } \\
\hline Pristolepis & marginata ${ }^{+}$Jerdon & HL, DY & $\mathrm{Ru}, \mathrm{PI}$ & $\mathrm{I}, \mathrm{m}$ & KLD, NEY \\
\hline \multicolumn{6}{|l|}{ Notopteridae } \\
\hline Notopterus & notopterus Pallas & $\mathrm{HL}$ & & & \\
\hline \multicolumn{6}{|l|}{ Osphronemidae } \\
\hline Pseudosphronemus & cupanus (Cuvier) & $\mathrm{HL}, \mathrm{DY}$ & & & \\
\hline \multicolumn{6}{|l|}{ Siluridae } \\
\hline Ompok & bimaculatus (Bloch) & HL, DY & $\mathrm{Ru}, \mathrm{PI}$ & $\mathrm{m}, \mathrm{h}$ & NEY \\
\hline Ompok & malabaricus ${ }^{+}$(Valenciennes) & HL, DY, EX & $\mathrm{Ru}, \mathrm{PI}$ & $\mathrm{m}, \mathrm{h}$ & NEY, KLD \\
\hline Wallago & attu Bloch \& Schneider & $\mathrm{HL}, \mathrm{DY}, \mathrm{OF}$ & & & \\
\hline \multicolumn{6}{|l|}{ Sisoridae } \\
\hline Glyptothorax & annandalei Hora & $\mathrm{HL}$ & & & \\
\hline Glyptothorax & Ionah Sikes & $\mathrm{HL}$ & & & \\
\hline Glyptothorax & madraspatanus + Day & $\mathrm{HL}$ & & & \\
\hline \multicolumn{6}{|l|}{ Synbranchidae } \\
\hline Monopterus & fossorius Nair & $\mathrm{HL}, \mathrm{OF}, \mathrm{DY}$ & & & \\
\hline \multicolumn{6}{|l|}{ Syngnathidae } \\
\hline Microphis & cuncalus Hamilton-Buchanan & $\mathrm{HL}$ & & & \\
\hline \multicolumn{6}{|l|}{ Tetraodontidae } \\
\hline Carinotetraodon & travancoricus ${ }^{+}$Hora \& Nair & $\mathrm{HL}, \mathrm{OF}$ & & & KLD \\
\hline
\end{tabular}

Key: Author names in brackets indicate redescriptions. Threats: HL - Habitat Loss; DY - Dynamite Fishing; OF - Overfishing; EX - Exotic species; IN Industrial Pollution. Preferred Habitat: Ru - Run; Ri - Riffle; Ra - Rapid; PI - Pool. Elevation range: I - low; m - mid; h - high. \# - Abraham et al. 2010; In Preparation; so still not a valid species. RE - Range extension to the Ashambu Hills Landscape; ^ - Taxonomy following new molecular study showing that the Indian species of Giant Snakehead; previously C. micropeltes should be treated as a distinct species C. diplogramma (Adamson et al. 2010).

Endemism: + - Western Ghats; ASH - Ashambu Hills. Occurrence in Rivers: KLD - Kallada; ITK - Ithikkara; VAM - Vamanapuram; KAR - Karamana; NEY - Neyyar. PA - Protected Area; NPA - Non-Protected Area.

collections where we thought it necessary, but avoided wanton collections on account of the threats faced by rare fishes even within Protected Areas. One of our important endeavours in this study has been to actively on taxonomic issues, avoid excessive collection for merely taxonomic work, especially from within conservations reserves and sanctuaries where many endemics occur (Abraham \& Kelkar, in Press) and also from unprotected areas. Many current and previous studies (e.g. Baby et. al. 2010) have employed the use of electro-fishing methods in critical aquatic habitats within conservation landscapes. We believe that there are and have to be more sensible ways, (although, of course, much more tedious and timeconsuming) for collection of fish species. Experienced fish taxonomists (such as Shri C. P. Shaji; pers.comm.) have observed mass mortality of several non-target aquatic species and life forms like amphibian tadpoles, juveniles fishes, crustaceans and macro-invertebrates, immediately following episodes of electro-fishing by 'scientific sampling' (Nielsen 1998).

We do not deny the importance of the respondent's suggestions. At the same time, we would like to stress the importance of minimally invasive ways for highly threatened taxa such as freshwater fishes and amphibians. We believe that the time's need is to go beyond mere stamp-collecting and check-listing, through inculcating certain conservation sensitivities in field research, and we are glad to have done that. We thank the respondent's thoughtful and in-depth comments on our article. Our revised checklist (Table 1) may be referred as an erratum to the original paper (Abraham et al. 2011). We also sincerely hope that this discussion would be useful for authors working on freshwater fishes in the region. 


\section{REFERENCES}

Abraham, R.K., N. Kelkar \& A.B. Kumar (2011). Freshwater fish fauna of the Ashambu Hills landscape, southern Western Ghats, India, with notes on some range extensions. Journal of Threatened Taxa 3(3): 1585-1593.

Abraham, R.K. \& N. Kelkar (2011 in press). Do terrestrial Protected Areas conserve freshwater fish diversity? Preliminary results from the southern Western Ghats, India. Oryx. (In Press - Manuscript accepted for publication).

Arnold, J.P. (1911). Der Formen- und Farbenkreis der Haplochilus panchax-Gruppe. Wochenschrift für Aquarienund Terrarienkunde 8(46): 669-672.

Arunachalam, M. \& J.A. Johnson (2002). A new species of Puntius. Hamilton (Pisces: Cyprinidae) from KalakadMundanthurai Tiger. Reserve, Tamil Nadu, India. Journal of the Bombay Natural History Society 99(3): 474-480.

Baby, F., J. Tharian, A. Ali \& R. Raghavan (2010). A checklist of freshwater fishes of the New Amarambalam Reserve Forest (NARF), Kerala, India. Journal of Threatened Taxa 2(12): 1330-1333.

Eschmeyer, W.N. \& J.D. Fong (2010). Species of fishes by family/subfamily. URL: <http://research.calacademy.org/ research/ichthyology/catalog/SpeciesByFamily.html>. Online version. Accessed 26 May 2010.

Eschmeyer, W.N. \& R. Fricke (eds.) (2011). Catalog of Fishes electronic version (29 March 2011). http://research calacademy.org/ichthyology/catalog/fishcatmain.asp. Accessed on 30th March 2011.

Euphrasia, C.J., K.V. Radhakrishnan \& B.M. Kurup (2006). The threatened freshwater fishes of Kerala, India. In: Kurup, B.M \& K. Ravindran (eds). Sustain Fish 2005, Proceedings of the International Symposium on improved sustainability of fish production systems and appropriate technologies for utilization. 16-18 March 2005, Kochi, India.

Jayaram, K.C. (1991). Revision of the Genus Puntius Hamilton. Records of the Zoological Survey of India Occasional Paper No. 135, Zoological Survey of India, Kolkata, India.

Jayaram, K.C. (2010). The Freshwater Fishes of the Indian Region. Narendra Publishing House, Delhi, 616pp.

Jerdon, T.C. (1849). On the fresh-water fishes of southern India. (Continued from p. 149.). Madras Journal of Literature and Science 15(2): 302-346.

Johnson, J.A. \& M. Arunachalam (2009). Diversity, distribution and assemblage structure of fishes in streams of southern Western Ghats, India. Journal of Threatened Taxa 1(10): 507-513.

Kurup, B.M., K.V. Radhakrishnan \& T.G. Manojkumar (2004). Biodiversity Status Of Fishes Inhabiting Rivers Of Kerala (S. India) With Special Reference To Endemism, Threats And Conservation Measures. URL: ftp://ftp.fao. org/docrep/fao/007/AD526e/ad526e12.pdf. Accessed on 25/4/2011

Nielsen, J.L. (1998). Scientific Sampling Effects: Electrofishing California's Endangered Fish Populations. Fisheries 23(12): 6-12.

Pethiyagoda, R.\& M. Kottelat (2005). The identity of the South Indian Barb Puntius mahecola (Teleostei: Cyprinidae), The Raffles Bulletin of Zoology 12: 145-152.

Raghavan, R. (2011). Need for further research on the freshwater fish fauna of the Ashambu Hills landscape: a response to Abraham et al. Journal of Threatened Taxa 3(5): 1788-1791. 